\title{
Role of Counterion Concentration in Determining Micelle Aggregation: Evaluation of the Combination of Constraints from Small-Angle Neutron Scattering, Electron Paramagnetic Resonance, and Time-Resolved Fluorescence Quenching
}

\author{
P. C. Griffiths* and A. Paul \\ School of Chemistry, Cardiff University, P.O. Box 912, Cardiff CF10 3TB, Wales, U.K. \\ R. K. Heenan and J. Penfold \\ Neutron Division, Rutherford and Appleton Laboratory, Chilton Didcot OX11 OQX, Oxon, U.K.
}

Radha Ranganathan and Barney L. Bales

Department of Physics and Astronomy and the Center for Supramolecular Studies, California State University, Northridge, California 91330-8268

Received: October 17, 2003; In Final Form: January 22, 2004

\begin{abstract}
Small-angle neutron scattering (SANS) has been used to study a series of aqueous solutions of (i) the anionic surfactant sodium dodecyl sulfate and the simple electrolyte sodium chloride and (ii) the cationic surfactants dodecyltrimethylammonium bromide and dodecyltrimethylammonium chloride, in the presence of sodium bromide and sodium chloride, respectively. For all three systems, the surfactant spans a wide concentration range, and by suitable choices of electrolyte concentration, it is possible to engineer these solutions to have the same concentration of counterions in the aqueous phase. According to a recent hypothesis by Bales et al. (J. Phys. Chem. B 2001, 105, 6798), such a series is expected to produce micelles having the same aggregation number $\left(N_{\text {agg }}\right)$, a point verified by time-resolved fluorescence quenching (TRFQ) and electron paramagnetic resonance (EPR). The SANS results presented here are in good agreement with the TRFQ and EPR studies. We therefore show unequivocally that the sizes and shapes of these surfactant micelles depend only on the free counterion concentration, a conclusion supported by conventional theories of micellization. Subsequently, for the SDS data, a comparison is made between fitting the SANS data to a model in which the aggregation number and degree of hydration are constrained and fitting them to a model in which these features are fittable parameters. In essence, both approaches yield the same conclusion. Such an approach is not possible with the cationic surfactants, as the contrast between the hydrated shell and the core is minimal.
\end{abstract}

\section{Introduction}

Small-angle neutron scattering (SANS) has evolved into a very powerful technique for studying the morphology of micelles formed in aqueous solution by surfactants, as well as the interaction between them. ${ }^{1,2}$ These properties are usually quantified within the context of a particular model describing the micelle morphology, as embodied by the form factor, and combined with some model taken from liquid theory to describe the intermicelle interactions via the structure factor.

The surfactant number or molecular packing parameter ${ }^{3}\left(N_{\mathrm{s}}\right)$ is a remarkably simple and insightful parameter to consider when discussing the morphology of the structures formed by surfactants. Defined as the ratio of the volume $(v)$ of the surfactant tail to the area per headgroup $\left(a_{0}\right)$ and the length of the surfactant tail $(l), N_{\mathrm{s}}=v / a_{0} l$, this dimensionless quantity can be translated into a specific micelle shape; that is, spherical micelles are predicted when $0<N_{\mathrm{s}}<1 / 3$, whereas, for cylindrical micelles, $1 / 3<N_{\mathrm{s}}<1 / 2$. Within the context of this study and established theories of micellization, an increase in the ionic strength of the solution will screen the interheadgroup repulsion, and thus, $a_{0}$ decreases. Given that $v$ and $l$ are constant, $N_{\mathrm{s}}$ increases and the micelle becomes more elongated. This simple approach suggests that there is a correlation between the ionic strength of the solution and the micelle morphology and, inter alia, the aggregation number.
The effective area per surfactant headgroup $\left(a_{0}\right)$ of an ionic surfactant micelle is a rather difficult quantity to calculate due to the tendency of the surfactant counterions to dissociate from the micelle surface. However, the dressed micelle model ${ }^{4,5}$ allows the degree of counterion dissociation $\left(\alpha_{\mathrm{Na}^{+}}\right)$to be calculated by solving the nonlinear Poisson-Boltzmann equation describing the ion distribution around a charged spherical structure. Typical values for SDS solutions $(16 \mathrm{mM}<$ [SDS] $<600 \mathrm{mM}$ in the presence of $0 \mathrm{mM}<[\mathrm{NaCl}]<200 \mathrm{mM}$ salt $)$ are $0.20<\alpha_{\mathrm{Na}^{+}}<0.30$, in excellent agreement with the same quantity measured by SANS. 6,23

Recently, the hypothesis was advanced ${ }^{7}$ that the aggregation numbers of ionic micelles, at a constant temperature, depend only on the concentration of counterions in the aqueous phase $\left(C_{\text {aq }}\right)$ :

$$
N_{\text {agg }}=N_{\text {agg }}\left(C_{\text {aq }}\right)
$$

Ionic micelles grow in response to increases in the value of $C_{\mathrm{aq}}$ whether the counterions are provided by the surfactant alone or by the surfactant plus any added electrolyte. ${ }^{7}$ Taking SDS and $\mathrm{NaCl}$ as an example, the micellized surfactant provides a counterion concentration equal to $\alpha_{\mathrm{Na}}+S_{\mathrm{m}}$, where $S_{\mathrm{m}}$ is the concentration of surfactant forming the micelles and $\alpha_{\mathrm{Na}^{+}}$is the degree of dissociation of the (sodium) counterions from the micelle surface. The concentration of counterions added via salt 
TABLE 1: Surfactant and Electrolyte Concentrations for the Samples Used in This Study, plus Corroborating Aggregation Numbers and Debye Lengths

\begin{tabular}{ccccccccc}
\hline$[\mathrm{SDS}] / \mathrm{M}$ & {$[\mathrm{NaCl}] / \mathrm{M}$} & $N_{\mathrm{TRFQ}}$ & $N_{\text {consensus }}{ }^{a}$ & $C_{\mathrm{aq}} / \mathrm{M}$ & $F\left(S_{\mathrm{t}}\right)$ & $\phi_{\mathrm{SDS}}$ & ionic strength/M & Debye length from $C_{\text {electrolyte }}, S_{\mathrm{f}}$, and $\alpha S_{\mathrm{m}} / \AA$ \\
\hline 0.660 & 0 & 111 & 112 & 0.188 & 1.236 & 0.19 & 0.09 & 10.0 \\
0.440 & 0.060 & 109 & 110 & 0.186 & 1.145 & 0.13 & 0.12 & 8.6 \\
0.330 & 0.090 & 108 & 110 & 0.188 & 1.105 & 0.095 & 0.13 & 8.1 \\
0.165 & 0.135 & 106 & 109 & 0.189 & 1.050 & 0.048 & 0.15 & 7.6 \\
0.085 & 0.157 & 107 & 108 & 0.186 & 1.026 & 0.025 & 0.15 & 7.4 \\
0.055 & 0.165 & 107 & 108 & 0.184 & 1.016 & 0.016 & 0.16 & 7.3
\end{tabular}

${ }^{a}$ Calculated using $\alpha_{\mathrm{Na}^{+}}=0.27$ and $\gamma=0.25$. If one uses $\alpha_{\mathrm{Na}^{+}}=0.24$ and $\gamma=0.20$, the aggregation numbers are larger by about 10 molecules.

is denoted $C_{\text {electrolyte. }} \mathrm{SDS}$ below the $\mathrm{cmc}$ and $\mathrm{NaCl}$ are strong electrolyes. Summing the contribution due to the monomeric ("free") surfactant $\left(S_{\mathrm{f}}\right)$, the micellized surfactant $\left(S_{\mathrm{m}}\right)$, and any added electrolyte yields a total counterion concentration in the aqueous phase of

$$
C_{\mathrm{aq}}=F\left(S_{\mathrm{t}}\right)\left[\alpha_{\mathrm{Na}^{+}} S_{\mathrm{t}}+\left(1-\alpha_{\mathrm{Na}^{+}}\right) S_{\mathrm{f}}+C_{\text {electrolyte }}\right]
$$

where $S_{\mathrm{t}}$ is the total concentration of surfactant and the factor

$$
F\left(S_{t}\right)=\frac{1}{(1-\phi)}
$$

takes into account the fact that the counterions do not penetrate the micelles. $\phi$ is the volume fraction occupied by the micelles and is commonly approximated to $\phi=V\left[S_{\mathrm{t}}\right]$, where $V$ is the molar volume of the surfactant, which assumes that the density of the unhydrated surfactant is approximately unity. In the SANS literature, $\phi$ is employed to correct for the penetrating background of the electrolyte. ${ }^{8}$ There are many combinations of $S_{\mathrm{t}}$ and $C_{\text {electrolyte }}$ that yield the same value of $C_{\text {aq }}$ and, according to eq 1 , the same aggregation number $\left(N_{\mathrm{agg}}\right)$. In the past, ${ }^{7}$ we have referred to the study of such a series of samples as a "constant $C_{\mathrm{aq}}$ experiment", although in practice such series are rarely strictly at a constant value of $C_{\mathrm{aq}}$ because of the factor $F\left(S_{\mathrm{t}}\right)$ and small density variations. ${ }^{7}$

The variation of $N_{\text {agg }}$ with $C_{\text {aq }}$ for SDS was found ${ }^{9}$ to follow the power law

$$
N_{\mathrm{agg}}=N^{\circ}\left(C_{\mathrm{aq}} / \mathrm{cmc}^{\circ}\right)^{\gamma}
$$

where $N^{\circ}=49.5, \gamma=0.25$, and $\mathrm{cmc}^{\circ}=0.0083 \mathrm{M}\left(\mathrm{cmc}^{\circ}\right.$ is the critical micelle concentration in the absence of salt)-values arrived at from data taken from a number of different experimental techniques. ${ }^{9}$ In eq 4 , to arrive at $C_{\text {aq }}$, a constant value of $\alpha_{\mathrm{Na}^{+}}=0.27$ was used. We call values of $N_{\mathrm{agg}}$ computed from eq 4 "consensus values". These values are given in Table 1.

Such a relationship is embodied in established theories on micellization but is rarely encountered in this guise. The free energy of micellization $\left(\Delta \mu_{N}^{\circ} / k T\right)$ can be calculated within various theoretical frameworks. ${ }^{10-12}$ For illustrative purposes, we use the conceptually simple approach proposed by Tanford, recently extended by Nagarajan, ${ }^{13}$

$$
\left(\frac{\Delta \mu_{N_{\text {agg }}}^{\circ}}{k T}\right)=\left(\frac{\Delta \mu_{N_{\text {agg }}}^{\circ}}{k T}\right)_{\text {transfer }}+\left(\frac{\Delta \mu_{N_{\text {agg }}}^{\circ}}{k T}\right)_{\text {interface }}+\left(\frac{\Delta \mu_{N_{\text {agg }}^{\circ}}^{\circ}}{k T}\right)_{\text {head }}
$$

where the subscripts refer to the free energy contribution of transferring the hydrophobic tail from an aqueous environment into the core of the micelle while leaving some of the hydrophobic core-the interface-exposed to the water phase and, concomitantly, introducing steric- and, for ionic surfactants, electrostatic repulsion between the neighboring headgroups.
Tanford then set

$$
\left(\frac{\Delta \mu_{N_{\mathrm{agg}}}^{\circ}}{k T}\right)_{\text {interface }}=\left(\frac{\sigma}{k T}\right) a_{\mathrm{e}}
$$

and

$$
\left(\frac{\Delta \mu_{N_{\mathrm{agg}}}^{\circ}}{k T}\right)_{\text {head }}=\left(\frac{\alpha}{k T}\right) \frac{1}{a_{\mathrm{e}}}
$$

where $\sigma$ is the interfacial tension, $\alpha$ is a headgroup repulsion parameter, and $a_{\mathrm{e}}$ is the equilibrium area of the hydrophobic core occupied by each surfactant molecule. Minimizing the free energy with respect to the aggregation number shows that only the second (interface) and third (head) terms contribute to determining the size and shape of the micelle-with the interface term decreasing and the head term increasing with a decrease in $a_{\mathrm{e}}$, equivalent to an increase in $N_{\text {agg. }}$.

For ellipsoidal aggregates, the equilibrium aggregation number is given by ${ }^{13}$

$$
N_{\mathrm{agg}}=\left(\frac{4 \pi l_{0}^{3}}{3 v_{0}}\right)\left(\frac{a_{\mathrm{e}} l_{0}}{v_{0}}-2\right)^{-1}
$$

Assuming a Debye-Hückel interaction between the ionic headgroups, the equilibrium area per molecule can be expressed as

$$
a_{\mathrm{e}}=\left(\frac{2 \pi e^{2} d}{\epsilon \sigma} \frac{1}{1+\kappa l_{0}}\right)^{2}
$$

where $d$ is the capacitor thickness, $\epsilon$ is the dielectric constant of the medium, $e$ is the charge of an electron, and $\kappa$ is the inverse Debye length. This last quantity has a square root relationship with ionic strength

$$
\kappa=\left(\frac{8 \pi e^{2}}{\epsilon k T} I\right)^{1 / 2}
$$

where $I$ is the ionic strength. By incorporating the approach embodied in eq 2 and the large particle limit to describe the double layer $\left(\kappa l_{0} \gg 1^{14,15}\right)$, it is clear that, since $a_{\mathrm{e}} \propto \kappa^{-2}$, then $N_{\text {agg }} \propto\left[\mathrm{Na}^{+}\right]^{1 / 4}$. In reality, $\kappa l_{0}$ is only slightly greater than 1 , and there may well be counterion-dependent steric or hydration factors present which would result in this simple approach not being exact. However, such a simple approach does describe quite a large body of data. ${ }^{7,16}$

In this study, we present SANS data from a series of SDS/ $\mathrm{NaCl}$, dodecyltrimethylammonium bromide (DTAB)/ $\mathrm{NaBr}$, and dodecyltrimethylammonium chloride (DTAC)/ $\mathrm{NaCl}$ systems for which we are able to predict the aggregation numbers and thus 
are able to test the prediction that the micelle morphology is invariant within this series of solutions studied.

\section{Experimental Section}

Materials. Sodium dodecyl sulfate (Aldrich) was recrystallized from ethanol until no dip in the surface tensionconcentration plot could be detected around the $\mathrm{cmc}$. All other reagents, including the cationic surfactants DTAB and DTAC, were of analytical grade and used as received. The solvent was $\mathrm{D}_{2} \mathrm{O}$ in the SANS and $\mathrm{H}_{2} \mathrm{O}$ in the time-resolved fluorescence quenching (TRFQ) and electron paramagnetic resonance (EPR) measurements, but this had no effect on the results.

Time-Resolved Fluorescence Quenching. TRFQ measurements were carried out on the SDS solutions with pyrene as the probe and dimethylbenzophenone as the quencher, both purchased from Aldrich and used as received. The samples were all prepared from one stock solution in a manner that allowed the relative values of the quencher/surfactant molar ratios to be determined with high precision. The average number of quenchers per micelle was constant. Therefore, any possible systematic errors that sometimes plague the interpretation of TRFQ data will be the same in all these samples, leading to better relative precision in the aggregation numbers. The fluorescence decay curves of pyrene were obtained at $25^{\circ} \mathrm{C}$ using an FL900 lifetime spectrometer (Edinburgh Analytical Instruments) and fit to the Infelta-Tachiya model ${ }^{17-19}$ using the software provided by the manufacturer. Before fitting, the decay curves were corrected for the instrument response. Details of the experimental setup and the procedure for calculating values of $N_{\text {agg }}$ are identical to those previously reported. ${ }^{20}$

Small-Angle Neutron Scattering. The SANS measurements were also performed as detailed previously ${ }^{21,22}$ on the fixedgeometry, time-of-flight LOQ diffractometer (ISIS Spallation Neutron Source, Oxfordshire, U.K.). All measurements were carried out at $25{ }^{\circ} \mathrm{C}$. Experimental measuring times were between 40 and $80 \mathrm{~min}$. All scattering data were normalized for the sample transmission and incident wavelength distribution and corrected for instrumental and sample backgrounds using an empty quartz cell and for the linearity and efficiency of the detector response. The data were put onto an absolute scale using a well-characterized partially deuterated polystyrene-blend standard sample.

SANS Data Fitting and Analysis. The intensity of scattered radiation $[I(Q)]$ as a function of the wave vector $(Q)$ is given by

$$
I(Q)=n_{\mathrm{m}}\left[S(Q)\langle F(Q)\rangle^{2}+\left\langle|F(Q)|^{2}\right\rangle-\langle F(Q)\rangle^{2}\right]+B_{\text {inc }}
$$

where $n_{\mathrm{m}}$ is the number of micelles per unit volume and $B_{\text {inc }}$ is the incoherent background scattering. The bracketed term, $[S(Q)$ $\left.\langle F(Q)\rangle^{2}+\left\langle|F(Q)|^{2}\right\rangle-\langle F(Q)\rangle^{2}\right]$, calculated from the decoupling approximation, ${ }^{23}$ describes the morphology of the scattering species, where $F(Q)=V_{1}\left(\rho_{1}-\rho_{2}\right) F_{0}\left(Q R_{1}\right)+V_{2}\left(\rho_{2}-\rho_{\text {solvent }}\right)$ $F_{0}\left(Q R_{2}\right)$. For an elliptical micelle, both $F(Q)$ and $F(Q)^{2}$ require numerical integration over an angle $\gamma$ between $Q$ and the axis of the ellipsoid to account for the random distribution of orientations of the ellipse. For clarity, we omit this. The first term represents the scattering from the core (subscript 1) of radius $R_{i}$ and axial ratio $X$, and the second, the polar shell (subscript 2). $V_{i}={ }^{4} / 3 \pi X R_{i}^{3}$ and $F_{0}\left(Q R_{i}\right)=3 j_{i}\left(Q R_{i}\right) / Q R_{i}\left(j_{i}\right.$ is the first-order spherical Bessel function of the first kind). The structure factor $S(Q)$ describes the spatial arrangement of the micelles in solution; this term is discussed in more detail below.

The structure factor is calculated from the RMSA approach on the basis of a repulsive Yukawa tail (an exponentially damped electrostatic term) and the hard-core potential. ${ }^{24,25}$ The model is described via four parameters: the hard sphere volume fraction ( $\left.\phi_{\text {hard sphere }}\right)$, the particle radius ( $\left.R_{\text {hard sphere }}\right)$, the micellar charge, and the inverse Debye screening length.

The hard sphere volume fraction is given by

$$
\phi_{\text {hard sphere }}=\frac{4 \pi N_{\mathrm{A}}\left(S_{\mathrm{t}}-S_{\mathrm{f}}\right) a^{3}}{3 N_{\mathrm{agg}}}
$$

where $a$ is the equivalent spherical outer radius of an ellipse, $a$ $=X^{1 / 3}\left(R_{\text {core }}+\delta_{\text {shell }}\right)$. The Debye length is calculated in the normal manner (eq 8), where the effective ionic concentration (strength) is taken as $2 S_{\mathrm{f}}+\alpha_{\mathrm{Na}}+\left(S_{\mathrm{s}}-S_{\mathrm{f}}\right)+2 C_{\text {electrolyte. Here, }}$ $\alpha_{\mathrm{Na}^{+}}$is obtained by dividing the surface charge by $N_{\text {agg }}$.

Scattering from Sodium Dodecyl Sulfate/Sodium Chloride Systems. Two approaches to parametrize the form factor for the SDS solutions have been undertaken. These are denoted "nonconstrained core-shell approach" and "EPR-TRFQ constrained core-shell approach".

Nonconstrained Core-Shell Approach. In this model, the inner core, with a radius equal to that of a fully extended dodecyl chain (16.7 $\AA$ ), contains only alkyl chains and is space filled. For aggregation numbers larger than $\sim 65$, the constraint that the minor radius of the core cannot be larger than $16.7 \AA$ results in the elongation of the micelle. The outer shell contains any alkyl chain not in the core, the sulfate headgroup, plus any counterions and hydration water. Therefore, the outer radius is also defined by space filling arguments. The degree of hydration is input in the form of a number of water molecules per surfactant headgroup, typically five. Thus, the $I(Q)$ is readily calculated using the decoupling approximation and the elliptical core - shell form factor described previously.

The only parameters refined in this model are the aggregation number $\left(N_{\mathrm{agg}}\right)$, the fraction of alkyl chain in the headgroup region, and the surface charge $(z)$, the parameters dependent on these (micelle ellipticity, ionic strength, and $R_{\text {hard sphere }}$ ) being recalculated at each iterative step of the fitting process. Here, it is found that roughly $10 \%$ of the alkyl chain is present in the headgroup, and thus, $V_{\text {hydrophobic tail }}=V_{\text {undecyl chain }}=323 \AA^{3}{ }^{26}$ equivalent to a bulk density of $0.80 \mathrm{~g} / \mathrm{mL}$ (compared to $\sim 0.75$ $\mathrm{g} / \mathrm{mL}$ for liquid dodecane). Implicit in this is the assumption that the methylene group closest to the sulfate group does not have a hydrophobic character. The volume of the headgroup $V_{\text {headgroup }}=74 \AA^{3}$, that is $60.6 \AA^{3}$ for the sulfate group and $13.6 \AA^{3}$ for the sodium ion. ${ }^{23}$

TRFQ Constrained Core-Shell Approach. The model of the micelle adopted here is also that of a charged particle with an elliptical core-shell morphology; however, the model is parametrized quite differently. In this case, all of the hydrocarbon chain is assumed to be present in the hydrophobic corehence, the minor radius of the ellipse is taken as the all-trans length of a $\mathrm{C}_{12}$ chain; that is, $R_{\text {core }}=16.7 \AA$. However, the aggregation number, $N_{\mathrm{agg}}=110$, is constrained to be that measured in the time-resolved fluorescence quenching experiment. Since all the methylenes are in the core of the micelle, multiplying this aggregation number by the volume for a dodecyl tail $\left(V_{\text {dodecyl tail }}=353 \AA^{3} ;^{32,27}\right.$ bulk density $\left.=0.8 \mathrm{~g} / \mathrm{mL}\right)$ provides the volume of the hydrophobic core $\left(V_{\text {core }}\right)$. Given the minor radius constraint, this inherently defines the micelle ellipticity as $X=2$; namely,

$$
V_{\text {core }}=V_{\text {dodecyl tail }} N_{\text {agg }}
$$




$$
X=\frac{3 V_{\text {core }}}{4 \pi R_{\text {core }}^{3}}
$$

In contrast to assigning a number of water molecules of hydration, as was done in the nonconstrained approach, the volume fraction of water in the shell $\left(\phi_{\mathrm{H}_{2} \mathrm{O}}\right)$ is constrained at the value determined from the EPR experiment; namely, $\phi_{\mathrm{H}_{2} \mathrm{O}}$ $=0.52 . V_{\text {sulfate(dry) }}$ is calculated assuming $\alpha_{\mathrm{Na}^{+}}=0.27$, that is, $60.6 \AA^{3}$ for the sulfate group and an average of $9.9 \AA^{3}$ for the bound sodium ions. Thus, the shell thickness $\left(\delta_{\text {shell }}\right)$ is also defined:

$$
V_{\text {shell }}=V_{\text {total }}-V_{\text {core }}=\frac{4 \pi}{3} X\left[\left(R_{\text {core }}+\delta_{\text {shell }}\right)^{3}-R_{\text {core }}^{3}\right]
$$

or

$$
V_{\text {shell }}=N_{\text {agg }} V_{\text {sulfate(dry) }}+\varphi_{\mathrm{H}_{2} \mathrm{O}} V_{\text {shell }}
$$

The core scattering length density was taken as that of dodecane $\left(\rho_{\text {core }}=-0.39 \times 10^{-6} \AA^{-2}\right)$. The scattering length density of the dry shell, that is $\rho_{\text {head }}$, was input as a constant ( $\rho_{\text {head }}=3.3 \times 10^{-6} \AA^{-2}$ ), but the fitting routine recalculates the scattering length density of the wet shell on the basis of the value of $\phi_{\mathrm{H}_{2} \mathrm{O}}$. We reiterate, like $V_{\text {sulfate(dry), }} \rho_{\text {head }}$ has been calculated by adopting $\alpha_{\mathrm{Na}^{+}}=0.27$. Hence, all parameters describing the form factor are essentially fixed. This constrained approach has been applied successfully to SDS/sugar surfactant ${ }^{21}$ and $\mathrm{SDS} / \mathrm{C}_{12} \mathrm{E}_{4}$ systems. $^{22}$

The approach adopted with the structure factor is similar to that of the nonconstrained model except that, since the aggregation number and degree of sodium ion dissociation $\left(\alpha_{\mathrm{Na}^{+}}\right)$are known, it should also be possible to constrain the ionic strength and the charge on the micelle (charge $=\alpha_{\mathrm{Na}^{+}} N_{\mathrm{agg}}$ ). Hence, in principle, all the parameters defining the $S(Q)$ routine can also be calculated. This contrasts with the nonconstrained approach, where $\alpha_{\mathrm{Na}^{+}}$(=charge/ $\left.N_{\mathrm{agg}}\right)$ is refined during fitting.

Scattering from Dodecyltrimethylammonium Bromide and Dodecyltrimethylammonium Chloride Systems. For the DTAB and DTAC systems, there is insufficient contrast between the core of the micelle $\left(\rho_{\text {core }}=-0.39 \times 10^{-6} \AA^{-2}\right)$ and the headgroup region $\left(\rho_{\text {head }}=-0.16 \times 10^{-6} \AA^{-2}\right.$ for DTAB and $\rho_{\text {head }}=-0.14 \times 10^{-6} \AA^{-2}$ for DTAC) to warrant fitting to a core-shell model. The scattering length densities of the headgroups are increased somewhat by the presence of water but not sufficiently to require a further step in the scattering length density profile. This small degree of hydration (compared to that of SDS) is consistent with the findings of Hayter and Penfold, ${ }^{23}$ who report a hydration number of $\sim 4$ for CTAB. The approach taken is otherwise identical; that is, the data were fit to a charged solid ellipse.

\section{Results}

Sodium Dodecyl Sulfate Solutions. Table 1 gives the values of $N_{\text {agg }}$ measured by TRFQ, and Figure 1 shows these values plotted against the consensus values. The values of $N_{\text {agg }}$ vary slightly because the samples detailed in Table 1 do not exactly form a constant $C_{\text {aq }}$ series, but they do agree with the consensus values extremely well. It is appropriate to note that although the absolute values of $N_{\text {agg }}$ are probably no better than $10 \%$, the relative values are quite precise because the relative quencher/surfactant ratios were precisely determined. Figure 1 reinforces an already large body of data $^{9}$ that substantiates the form of eq 4 and the simple theoretical approach given previously.

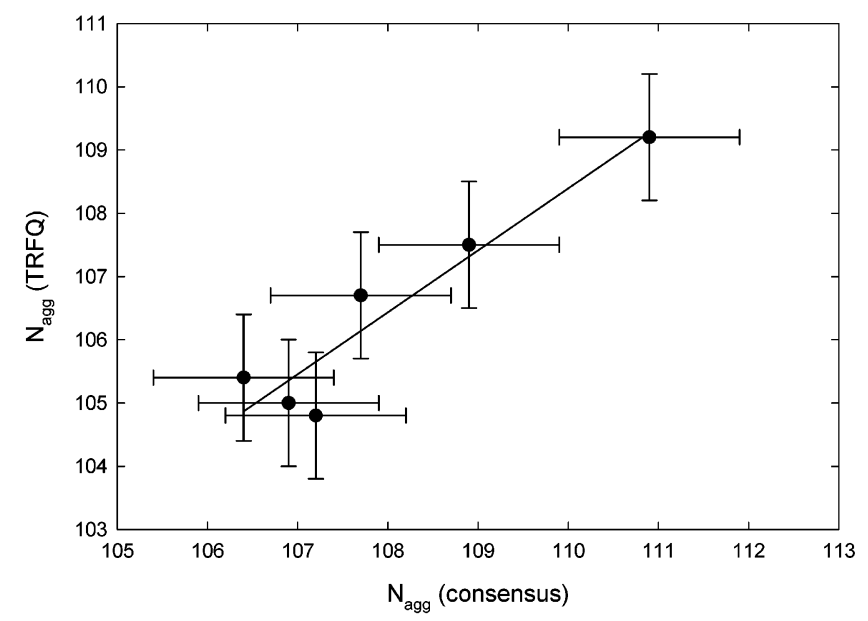

Figure 1. Values of $N_{\text {agg }}$ for SDS micelles measured by TRFQ plotted against the consensus values. The values of $N_{\text {agg }}$ vary slightly because the samples detailed in Table 1 do not exactly form a constant $C_{\text {aq }}$ series.

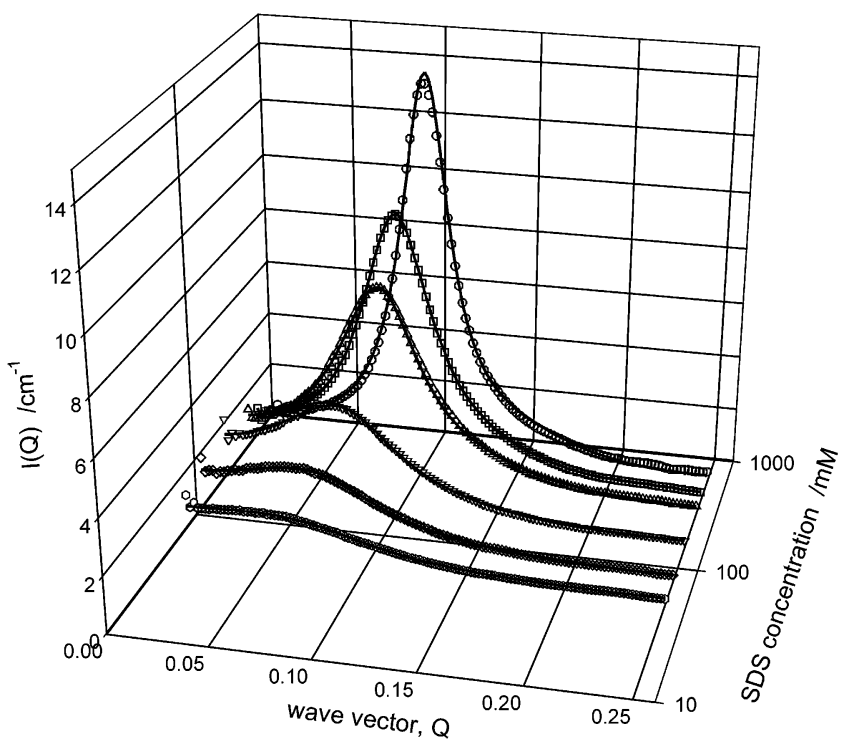

Figure 2. Raw SANS data for $20<$ [SDS] $<660 \mathrm{mM}$ with varying $[\mathrm{NaCl}]$. The data become more intense, with the structure peak moving toward a high $Q$, with increasing [SDS]. The solid lines correspond to the fits to the two models as described in the text.

The raw SANS data are shown in Figure 2; with increasing surfactant concentration, the peak due to the structure factor becomes increasingly more pronounced and moves to a higher $Q$, associated with the decreasing separation of the surfactant micelles. Over this series, $N_{\text {agg }}=108 \pm 3$; that is, it is effectively constant throughout the series. The only difference between the various samples is the number of micelles present (eq $9 ; n_{\mathrm{m}}$ ). Therefore, the SANS data should be described by a common set of parameters, with only $n_{\mathrm{m}}$ and $S(Q)$ (by virtue of $n_{\mathrm{m}}$ ) varying between samples.

Two approaches to fitting these data have been tested. The fits are indistinguishable, and both fit the data remarkably well (Figure 2). The parameters are presented in Table 2 for the nonconstrained approach and Table 3 for the EPR-TRFQ constrained approach. As may be seen from both approaches, the main point of this study has been verified, namely that the micelle size and shape are invariant (within experimental error), reflecting the importance of the aqueous phase sodium ion counterion concentration $\left(C_{\mathrm{aq}}\right)$, rather than either the total surfactant concentration $\left(S_{\mathrm{t}}\right)$ or the concentration of added electrolyte $\left(C_{\text {electrolyte }}\right)$, in determining micelle morphology. 
TABLE 2: Parameters Describing the Fits to the Nonconstrained Model

\begin{tabular}{|c|c|c|c|c|c|c|c|c|c|c|c|}
\hline$[\mathrm{SDS}] / \mathrm{M}$ & {$[\mathrm{NaCl}] / \mathrm{M}$} & $N_{\text {agg }}$ & $\begin{array}{c}\text { surface charge } \\
(z)\end{array}$ & $\begin{array}{l}\text { Debye } \\
\text { length/Å }\end{array}$ & $\begin{array}{c}\text { inner radius } \\
\left(R_{1}\right) / \AA\end{array}$ & $\begin{array}{l}\text { outer radius } \\
\left(R_{2}\right) / \AA\end{array}$ & $\begin{array}{l}\text { ellipticity } \\
\qquad(X)\end{array}$ & \multicolumn{2}{|c|}{ degree of hydration ${ }^{a}$} & $\alpha_{\mathrm{Na}^{+}}$ & $R_{\text {hard sphere }}$ \\
\hline 0.660 & 0 & 136 & 33 & 10.0 & 16.7 & 19.8 & 2.28 & 5 & 0.67 & 0.24 & 26.1 \\
\hline 0.440 & 0.060 & 137 & 31 & 9.1 & & & 2.30 & & 0.69 & 0.23 & 25.7 \\
\hline 0.330 & 0.090 & 132 & 33 & 8.3 & & & 2.20 & & 0.70 & 0.25 & 25.2 \\
\hline 0.165 & 0.135 & 123 & 28 & 8.3 & & & 1.96 & & 0.68 & 0.23 & 24.9 \\
\hline 0.085 & 0.157 & 120 & 26 & 7.7 & & & 2.00 & & 0.70 & 0.22 & 24.2 \\
\hline 0.055 & 0.165 & 114 & 40 & 7.7 & & & 1.92 & & 0.70 & 0.35 & 24.2 \\
\hline
\end{tabular}

${ }^{a}$ The number of water molecules per surfactant headgroup.

TABLE 3: Parameters Describing the Fits to the EPR-TRFQ Constrained Model

\begin{tabular}{|c|c|c|c|c|c|c|c|c|c|c|c|}
\hline$[\mathrm{SDS}] / \mathrm{M}$ & {$[\mathrm{NaCl}] / \mathrm{M}$} & $N_{\text {agg }}$ & $\begin{array}{c}\text { surface charge } \\
(z)\end{array}$ & $\begin{array}{r}\text { Debye } \\
\text { length/Å }\end{array}$ & $\begin{array}{c}\text { inner radius } \\
\left(R_{1}\right) / \AA\end{array}$ & $\delta_{\text {shell }} / \AA ̊ ̊$ & $\begin{array}{l}\text { ellipticity } \\
\qquad(X)\end{array}$ & $\begin{array}{c}\text { degree of } \\
\text { hydration }^{a}\end{array}$ & $\alpha_{\mathrm{Na}^{+}}$ & $R_{\text {hard sphere }}$ & $\phi_{\text {hard sphere }}$ \\
\hline 0.660 & 0 & 110 & 35 & 9.3 & 16.7 & 2.6 & 2.00 & 0.52 & 0.32 & 26.0 & 0.190 \\
\hline 0.440 & 0.060 & & 37 & 8.3 & & & & & 0.34 & 25.8 & 0.127 \\
\hline 0.330 & 0.090 & & 35 & 8.0 & & & & & 0.32 & 25.4 & 0.095 \\
\hline 0.165 & 0.135 & & 36 & 7.6 & & & & & 0.33 & 24.4 & 0.048 \\
\hline 0.085 & 0.157 & & 38 & 7.4 & & & & & 0.34 & 21.8 & 0.025 \\
\hline 0.055 & 0.165 & & 32 & 7.3 & & & & & 0.30 & 20.0 & 0.016 \\
\hline
\end{tabular}

${ }^{a}$ The amount of water in the polar shell expressed as a volume fraction.

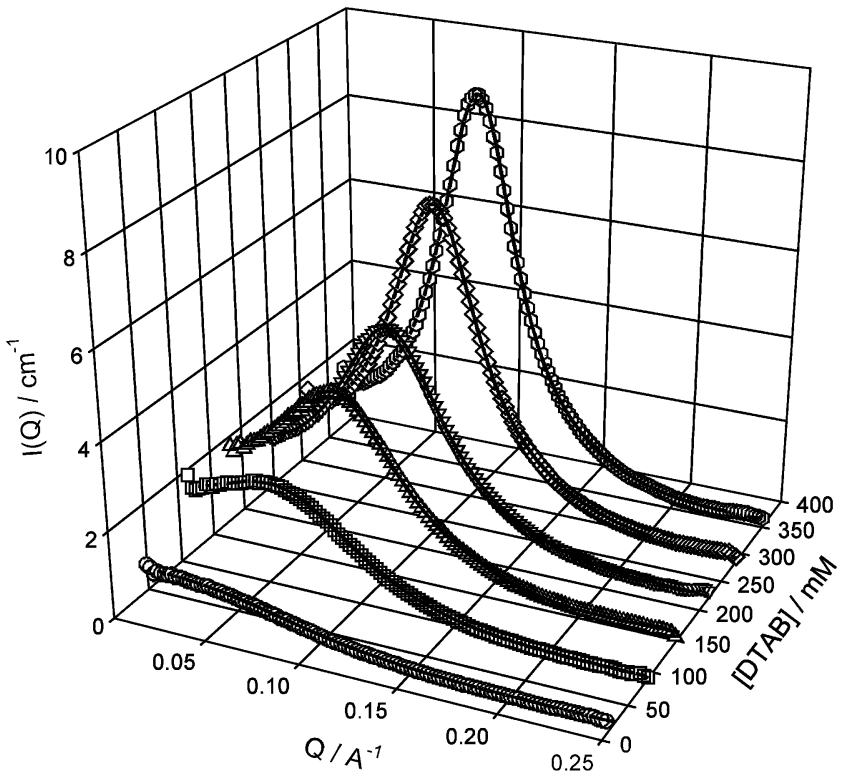

Figure 3. SANS from a constant $C_{\text {aq }}$ series of DTAB with $\mathrm{NaBr}$. Lines are best fits to the data based on a charged, solid ellipse model. Concentrations were as follows $[\mathrm{DTAB} / \mathrm{mM}] /[\mathrm{NaBr} / \mathrm{mM}]$ : circles, $25 /$ 95; squares, 90/76; triangles, 155/57; inverted triangles, 220/38; diamonds, 285/19; hexagons, 350/0.

All these data are best fitted using ellipses, rather than polydisperse spheres. For the nonconstrained model, minor adjustments to the model do not improve the fits; that is, changing the hydration per headgroup from 0 to 10 , although 5 is mostly used, does not alter the model parameters or the quality of the fit. This is in good agreement with the conclusion reached in the constrained fitting approach. Since $10 \%$ uncertainties in the scale factor, aggregation number, and surface charge are acceptable, there is agreement with the central premise of this paper-namely, that, over the whole range of the data, the aggregation number is largely invariant.

DTAB and DTAC Solutions. The question then arises as to whether or not this is a general behavior or specific to SDS. Presented in Figures 3 and 4 are analogous data and fits for the $\mathrm{DTAB} / \mathrm{NaBr}$ and DTAC/NaCl solutions. The essential parameters are given in Tables 4 and 5. As can be seen, the aggregation numbers thus obtained are consistent with those found by TRFQ $(73 \pm 2$ DTAB, $60 \pm 2$ DTAC). Since there is no shell in this

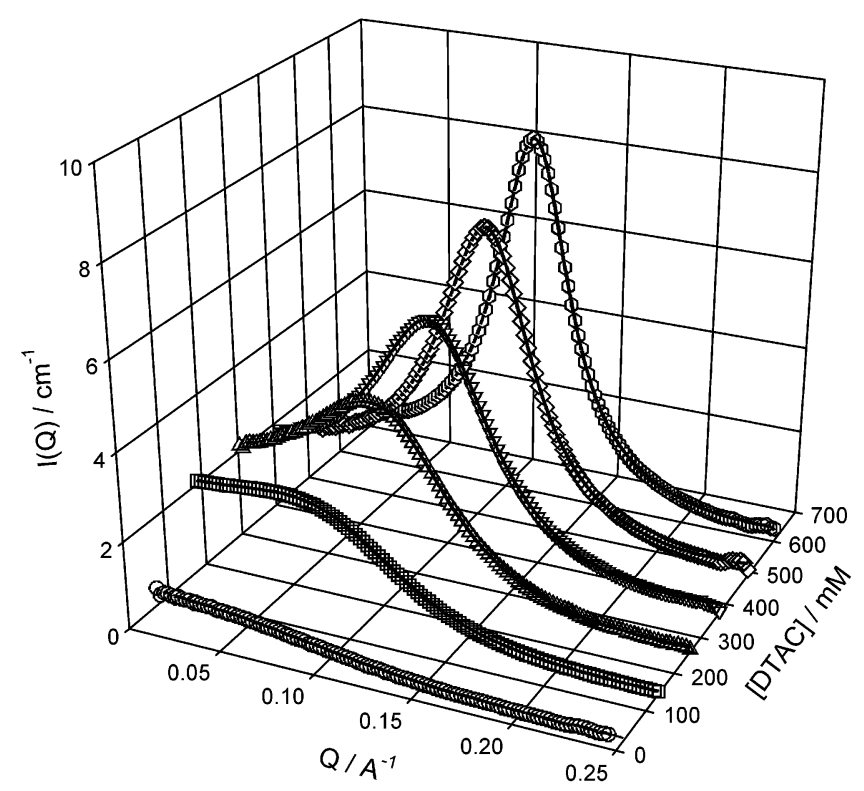

Figure 4. SANS from a constant $C_{\text {aq }}$ series of DTAC with $\mathrm{NaCl}$. Lines are best fits to the data based on a charged, solid ellipse model. Concentrations were as follows [DTAC/mM] $/[\mathrm{NaCl} / \mathrm{mM}]$ : circles, 32/ 251; squares, 144/201; triangles, 258/151; inverted triangles, 372/100; diamonds, 486/50; hexagons, 600/0.

TABLE 4: Principal Parameters Describing the Fits to the SANS Data Using a Charged Solid Ellipse

(Dodecyltrimethylammonium Bromide/Sodium Bromide Solutions)

\begin{tabular}{ccccc}
\hline$[\mathrm{DTAB}] / \mathrm{M}$ & {$[\mathrm{NaBr}] / \mathrm{M}$} & $\begin{array}{c}R / \AA \\
( \pm 0.2)\end{array}$ & ellipticity $(X)$ & $\begin{array}{c}N_{\text {agg }} \\
( \pm 5)^{a}\end{array}$ \\
\hline 0.025 & 0.095 & 18.2 & 1.7 & 71 \\
0.090 & 0.076 & 17.5 & 1.9 & 70 \\
0.155 & 0.057 & 18.0 & 1.9 & 77 \\
0.220 & 0.038 & 17.4 & 1.8 & 66 \\
0.285 & 0.019 & 17.7 & 1.8 & 69 \\
0.350 & 0 & 18.0 & 1.9 & 74 \\
${ }^{a} \mathrm{~N}_{\text {agg }}$ (TRFQ) & $=73$. & & &
\end{tabular}

model, the radius is in fact the outer radius of the micelle, that is, $R_{\text {core }}+\delta_{\text {shell. }}$. The freely fitted data arrive (within experimental error) at the same ellipticity and radii obtained from the TRFQ calculation. This simpler approach has again arrived at the similar conclusion found previously-that the aggregation 
TABLE 5: Principal Parameters Describing the Fits to the SANS Data Using a Charged Solid Ellipse

(Dodecyltrimethylammonium Chloride/Sodium Chloride Solutions)

\begin{tabular}{ccccc}
\hline [DTAC]/M & {$[\mathrm{NaCl}] / \mathrm{M}$} & $\begin{array}{c}R / \AA \\
( \pm 0.2)\end{array}$ & ellipticity $(X)$ & $\begin{array}{c}N_{\text {agg }} \\
( \pm 5)^{a}\end{array}$ \\
\hline 0.030 & 0.251 & 17.5 & 1.4 & 63 \\
0.144 & 0.201 & 18.0 & 1.4 & 69 \\
0.258 & 0.151 & 18.1 & 1.3 & 65 \\
0.372 & 0.100 & 17.4 & 1.4 & 62 \\
0.486 & 0.050 & 17.5 & 1.4 & 64 \\
0.600 & 0 & 17.5 & 1.4 & 62 \\
${ }^{a} \mathrm{~N}_{\text {agg }}($ TRFQ $)$ & $=60$. & & &
\end{tabular}

number and hence micelle size and shape are indeed a function of the solution counterion concentration only.

\section{Discussion}

The SANS and TRFQ results presented here substantiate the previously presented EPR data on the constant $C_{\text {aq }}$ series for SDS; the micelle aggregation number is largely invariant with the $[\mathrm{SDS}] .^{7}$ The SANS and TRFQ results presented here for the cationic surfactants suggest that this is a general phenomenon.

It is appropriate to review the essence of the three methods in their approach to studying $N_{\text {agg }}$. The TRFQ technique essentially "counts" the number of micelles. The technique involves several possible sources of systematic error. First, the value of $N_{\mathrm{agg}}$ can only be as accurate as the value of the quencher concentration. Second, the value of $S_{\mathrm{f}}$ must be subtracted from $S_{\mathrm{t}}$ in the analysis. Third, the statistical distribution of the quenchers among the micelles, usually assumed to be Poissonian, must be known. Fourth, the effect of polydispersity has never been dealt with satisfactorily despite much effort. All of these uncertainties can add up to an uncertainty in the absolute value of $N_{\text {agg }}$. Nevertheless, TRFQ does very well indeed when compared with the consensus value (eq 4) derived from various techniques. The same conclusion is supported in many studies involving other micelles. ${ }^{28}$ More important in the context of this work is the fact that TRFQ is even more reliable as an indicator of relative values of $N_{\mathrm{agg}}$, since the systematic errors due to the four uncertainties just mentioned will be very nearly the same in every member of the sample series. Further, the values of unimer concentrations for all members of this series are so small compared with the values of $S_{\mathrm{t}}$ that even a large uncertainty in $S_{\mathrm{f}}$ would be completely negligible.

The EPR approach to studying $N_{\text {agg }}$ is very different. Here, we do not determine the value of $N_{\text {agg }}$; we ascertain, to a high precision, if the micelles in two samples have the same value of $N_{\text {agg. }}$. This is accomplished using techniques exhaustively detailed in recent publications. ${ }^{29-32}$ In essence, a spin-probe is solubilized by the micelles in low concentration and the polarity sensed by the probe is from the hyperfine coupling. Similar work $^{28-31}$ has related the measured polarity to the hydration of the micelle surface. However, in the present context, it is not necessary to invoke that model or even that the model be correct. All that is required is that the spin-probe senses the same polarity if the micelle is the same size. This has been amply demonstrated and is expected from what is known ${ }^{33}$ about the mechanism of the effect of local electric fields on the hyperfine coupling. The only conceivable ways for the hyperfine coupling constant to be different in two micelles of the same size would be if the spin-probe senses the nearest neighbor micelles or if the neighbors induce a change in the average location of the spin-probe. Both of these possibilities are very unlikely.

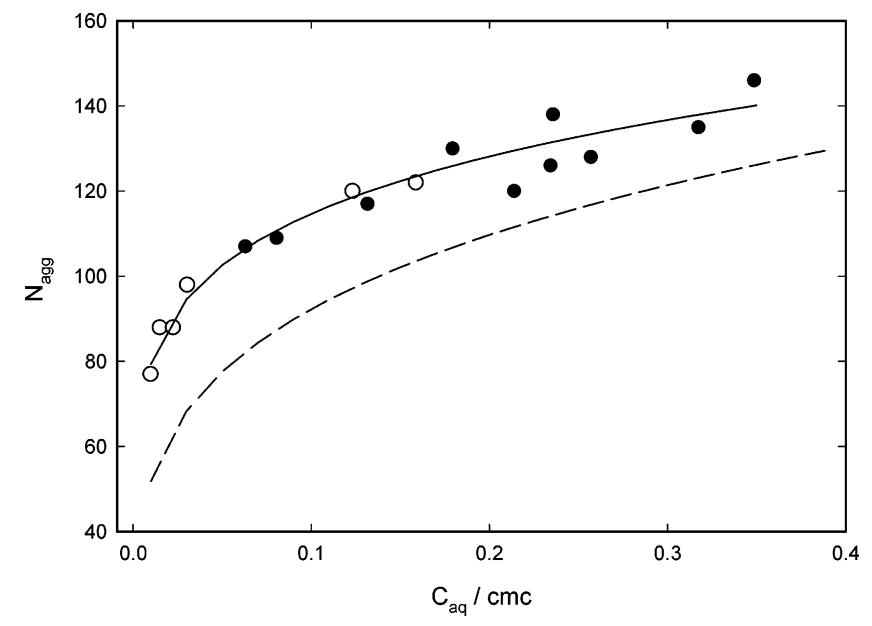

Figure 5. Aggregation number extracted from the previous work of Hayter and Penfold ${ }^{23}$ plotted as a function of the free sodium ion concentration normalized to the critical micelle concentration. The open symbols correspond to no added salt; the closed symbols correspond to added salt. The broken line represents the concensus values used here; the solid line represents the behavior outlined in the text with the exponent $\alpha_{\mathrm{Na}^{+}}=0.16$.

The SANS method senses the size and to a lesser extent the shape of the micelles, but a detail such as $N_{\text {agg }}$ is usually extracted within the context of a model. Thus, it is very different from the previous two methods. This model dependence is a weakness of SANS. Further, unlike the cases of the two previous methods, the details of the distribution of the micelles and their concentrations enter directly into the analysis.

Despite this apparent weakness, SANS studies support the constant conclusion that $N_{\text {agg }}$ depends only on the sodium ion concentration. Consider only the $\mathrm{SDS} / \mathrm{NaCl}$ systems, for which there is a larger body of data available compared to the case of the cationics. Previous SANS experiments are in agreement with the form of eq 4 (Figure 5), albeit with slightly different parameters, $N_{\mathrm{agg}}=164 C_{\mathrm{aq}}{ }^{0.16}$, and marginally larger aggregation numbers. Clearly, the raw data fit the power law well and describe the growth of SDS micelles whether the concentration of surfactant or salt is increased. Note the abscissa is computed supposing a constant value of $\alpha_{\mathrm{Na}^{+}}=0.27$, as found previously. The solid line below the SANS data gives the consensus values of $N_{\text {agg }}$, that is, those extracted from a variety of studies..$^{9}$ Our (TRFQ) value of $N_{\text {agg }}=110$ falls on this latter line. The limited data on the cationics also support this approach. Thus, all three methods have their weaknesses. Insisting that a given model be consistent with all three methods is the best insurance against misinterpretation of any one of the three. We test the viability of such an approach using the SDS data.

Consider now the detail within the fitting, implications of invoking constraints, and the coarse grained nature of the model. Effectively, the data analysis involves fitting a step-profile to the scattering length density cross section of the micelle. The core of the micelle, consisting of (hydrogenous) hydrocarbon chains, has a scattering length density of $\sim-0.56 \times 10^{-6} \AA^{-2}$. For a shell consisting of hydrated headgroups, the scattering length density is rather similar to that of the solvent, $\sim 4.0 \times$ $10^{-6} \AA^{-2}$, essentially due to the degree of water present in the headgroup region. The continuous phase has a quite different scattering length density, $\sim 6.4 \times 10^{-6} \AA^{-2}$. The principle contrast step is, therefore, at the core/shell interface. Indeed, simply fitting these data to a solid ellipse returns a fit that is remarkably similar to the core dimensions. The core-shell approach forces the fitting routine to include a "step" in the scattering length density cross section of the micelle. The model 
then parametrizes the width and height of this step. Inherently, the fitting routine is less sensitive to this step, and we invoke various criteria (core radius, ellipticity, etc.) to limit the fitting. The key point to remember, of course, is that the micelle is a dynamic structure and there will be no sharp discontinuities in scattering length density between the core, shell, and continuous phases. In this regard, the model can introduce artifacts and it is these that we aim to guard against.

With that in mind, let us compare and contrast the two approaches. Both models confirm that the gross morphology of the micelle is the same-elliptical micelles with an axial ratio of 2 and a core radius of $16.7 \AA$.

The main difference between the two models is how the volume of the hydrophobic tail is incorporated into the calculation of the scattering length density profile and, therefore, the aggregation number and counterion binding. The constrained model uses a volume of $353 \AA^{3}$ which assumes that all 12 carbons of the alkyl group are in the hydrophobic core, whereas the nonconstrained model effectively only includes 11 in the core and therefore uses a volume of $323 \AA^{3}$. Ostensibly, this seems to be a significant discrepancy, and it might be that this delineation of the core/shell interface leads to the overestimation of the aggregation number within the nonconstrained approach (since the micelles in both models have the same physical size for the core, $16.7 \AA$ ).

An important characteristic of the micelle is its effective charge, expressed as the degree of counterion binding $\left(\alpha_{\mathrm{Na}^{+}}{ }^{+}\right.$, which may be obtained from the SANS analysis as charge $/ N_{\text {agg }}$. The original SANS analysis of SDS by Hayter and Penfold ${ }^{23}$ yielded $\alpha_{\mathrm{Na}^{+}}=0.23 \pm 0.05$, in excellent agreement with the theoretical value calculated from the dressed micelle model, ${ }^{4}$ $\alpha_{\mathrm{Na}^{+}}^{\mathrm{DMT}}=0.24 \pm 0.03$. The nonconstrained analysis of the current SDS data suggests $\alpha_{\mathrm{Na}^{+}}=0.25 \pm 0.05$. Thus, the two experiments show good mutual agreement. For the constrained fitting approach applied to the current data, slightly larger estimates of $\alpha_{\mathrm{Na}^{+}}$are obtained: $\alpha_{\mathrm{Na}^{+}}=0.33 \pm 0.02$, while $\alpha_{\mathrm{Na}^{+}}^{\mathrm{DMT}}=0.27 \pm 0.03$.

Thus, it would appear that the constrained model, compared to the nonconstrained model at least, somewhat overestimates the degree of counterion dissociation, although the calculated quantity is within experimental error of the theoretical value. However, the constrained approach inherently returns the correct aggregation number. These discrepancies are, in reality, mute points, as they are far more sensitive to the volume of the hydrophobic tail and how it is incorporated into the model of the micelle.

\section{Conclusions}

We show unequivocally that the size, shape, and aggregation number of SDS, DTAB, and DTAC micelles depend only on the free counterion concentration, a quantity that depends on the concentration of both the surfactant and any added salt.
For SDS solutions, a number of quite different techniquesSANS, EPR, and TRFQ-have been combined to verify the SANS model and evaluate the constrained fitting used in the SANS analysis. Ultimately, this has the result of slightly decreasing the perceived aggregation number extracted from the SANS analysis but at the expense of a larger error associated with the counterion binding. A simpler approach adopted with DTAB and DTAC gives the same conclusions.

Acknowledgment. We gratefully acknowledge support from NIH/MBRS S06 GM48680-03 and the EPSRC for provision of SANS beam time.

\section{References and Notes}

(1) Penfold, J. Encyclopedia of Surface and Colloid Science; Marcel Dekker: 2002; p 3653.

(2) Pedersen, J. G. Curr. Opin. Colloid Interface Sci. 1999, 4, 190.

(3) Israelachvili, J.; Mitchell, D. J.; Ninham, B. W. J. Chem. Soc., Faraday Trans. 1976, 72, 1525.

(4) Evans, D. F.; Mitchell, D. J.; Ninham, B. W. J. Phys. Chem. 1984, $88,6344$.

(5) Hayter, J. B. Langmuir 1992, 8, 2873.

(6) Hayter, J. B.; Zemb, T. N. Chem. Phys. Lett. 1982, 93, 91.

(7) Bales, B. L. J. Phys. Chem. B 2001, 105, 6798.

(8) Snook, I. K.; Hayter, J. B. Langmuir 1992, 8, 2880.

(9) Quina, F. H.; Nassar, P. M.; Bonilha, J. B. S.; Bales, B. L. J. Chem. Phys. 1995, 99, 17028.

(10) Nagarajan, R.; Ruckenstein, E. Langmuir 1991, 7, 2934.

(11) Tanford, C. The Hydrophobic Effect; Wiley-Interscience: New York, 1973.

(12) Shiloach, A.; Blankschtein, D. Langmuir 1998, 14, 7166.

(13) Nagarajan, R. Langmuir 2002, 18, 31.

(14) Griffiths, P. C.; Pettersson, E.; Stilbs, P.; Cheung, A. Y. F.; Howe, A. M.; Pitt, A. R. Langmuir 2001, 17, 7178.

(15) Griffiths, P. C.; Paul, A.; Stilbs, P.; Pettersson, E. Langmuir 2003, 19,8605 .

(16) Bergström, M.; Pederson, J. S. Phys. Chem. Chem. Phys. 1999, 1, 4437.

(17) Gehlen, M. H.; De Schryver, F. C. Chem. Rev. 1993, 93, 199.

(18) Infelta, P. P.; Grätzel, M.; Thomas, J. K. J. Phys. Chem. 1974, 78, 190.

(19) Tachiya, M. Chem. Phys. Lett. 1975, 33, 289.

(20) Ranganathan, R.; Tran, L.; Bales, B. L. J. Phys. Chem. B 2000, $104,2260$.

(21) Griffiths, P. C.; Finney, G.; Cheung, A. F. Y.; Howe, A. M.; Pitt, A. R.; King, S. M.; Heenan, R. K.; Bales, B. L. Langmuir 2002, 18, 1065.

(22) Griffiths, P. C.; Cheung, A. Y. F.; Farley, C.; Paul, A.; Heenan, R. K. J. Phys. Chem. B, in press.

(23) Hayter, J. B.; Penfold, J. Colloid Polym. Sci. 1983, 261, 1022.

(24) Hansen, J. P.; Hayter, J. B. Mol. Phys. 1982, 46, 651.

(25) Hayter, J. B.; Penfold, J. Mol. Phys. 1981, 42, 109.

(26) Tanford, C. J. Phys. Chem. 1974, 78, 2469.

(27) Nagarajan, R. J. Chem. Phys. 1989, 90, 1980.

(28) Bales, B. L. J. Phys. Chem. B 2001, 105, 6798

(29) Bales, B. L.; Messina, L.; Vidal, A.; Peric, M.; Nascimento, O. R. J. Phys. Chem. B 1998, 102, 10347.

(30) Bales, B. L.; Shahin, A.; Lindblad, C.; Almgren, M. J. Phys. Chem. $B$ 2000, 104, 256 .

(31) Bales, B. L. J. Phys. Chem. B 2001, 105, 6798.

(32) Bales, B. L.; Zana, R. J. Phys. Chem. B 2002, 106, 1926.

(33) Schwartz, R. N.; Peric, M.; Smith, S. A.; Bales, B. L. J. Phys. Chem. B 1997, 101, 8735. 